International Journal of Biomedicine I June 2019 - Volume 9, Issue Suppl_1: Abstracts From the Second Russian International Conference "Cryo-electron microscopy 2019: achievements and prospects"

ORAL ABSTRACT PRESENTATIONS

SESSION TITLE: COMPLEX AND EMERGING TECHNIQUES IN STRUCTURAL BIOLOGY

DOI: 10.21103/IJBM.9.Suppl_1.OR23

\title{
Abstract OR-23: Current Status of Biomolecules Imaging by X-ray Free Electron Laser without Crystallization
}

\author{
Aleksandr V. Kudriavtsev ${ }^{1,2}$, Anna V. Vakhrusheva ${ }^{1}$, Valery N. Novoseletsky ${ }^{1}$, Grigoriy A. Armeev ${ }^{1}$, \\ Mikhail A. Lozhnikov ${ }^{1}$, Alexey K. Shaitan ${ }^{1}$, Georgy M. Kobelkov ${ }^{1}$, Konstantin V. Shaitan ${ }^{1}$ \\ ${ }^{1}$ Lomonosov Moscow State University, Faculty of Biology, Moscow, Russia \\ ${ }^{2}$ Emanuel Institute of Biochemical Physics, Russian Academy of Sciences, Moscow, Russia
}

Background: X-ray free-electron lasers (XFELs) in structural biology field opened a new opportunity for studying the structural dynamics of biomolecules. Structural dynamics analysis of single protein molecules by XFEL is based on the "diffraction-before-destruction" principle. The obtaining of the diffraction without sample crystallization is a complicated. Despite this fact, there are works about studying of single biomolecules without crystallization by XFEL.

Methods: We have analyzed some works from the Coherent X-ray Imaging Data Bank (http://www.cxidb.org). Among all presented structures the works, that contained biological object and did not include crystallization methods for its preparing, were selected. Only the following methods Single Particle Flash X-ray Imaging (SP-XDI), Serial Fiber Diffraction (SFD), Fluctuation X-ray Scattering (FXS) met the chosen criteria. Studies with different objects were considered as different works.

Results: In total, 20 non-recurring studies about studying of various biological objects without usage of crystallization were found. Among them there were two works with usage of FXS method, three works about SFD method and the remaining 15 works - with SP-XDI method. The works were also classified by objects: three studies were about cell structure of yeast and cyanobacteria; in two cases the structures of fibrils (bombesin and endorphin) were investigated by SFD method; in two other papers - the structures of protein complexes (RNA Polymerase II and Carboxysomes); and in the remaining 13 articles the viruses of various size were studied. Based on the analysis of works the potential problems of this field were elucidated, such as processing of diffraction patterns and 3D-reconstruction of structures, preparation of small size objects (single proteins less than $500 \mathrm{kDa}$ ), and low ratio between successful and error hits.

Conclusion: Currently, the field of structural biology has gained necessary conditions for the analysis of single biological molecules. It was noted in the recent study (Pietrini, 2018), in case of solution of such problems as reducing electrospray aerosol droplet size, will allow to get the $5 \AA$ 
resolution. Thus, it makes the problem of 3D-reconstruction from XFEL diffraction patterns is extremely important area for future studies.

Key Words: XFEL • single molecules $\bullet$ diffraction

Sources of Funding: This work was supported by the Russian Foundation for Basic Research No. 18-0240010.

International Journal of Biomedicine. 2019;9 Suppl 1: S15. doi: 10.21103/IJBM.9.Suppl_1.OR23

C2019 International Medical Research and Development Corporation 